\title{
Trial of a long-acting somatostatin analogue for autosomal dominant polycystic kidney disease
}

Treatment of patients with autosomal dominant polycystic kidney disease (ADPKD) with a long-acting somatostatin analogue for 3 years slows kidney volume and kidney cyst growth, according to new data from a multicentre, randomized, single-blind, placebo-controlled trial. "The finding that the rate of total kidney volume growth averaged throughout the study was about $50 \%$ slower in patients receiving the long-acting somatostatin analogue than in patients receiving placebo provides convincing evidence of a sustained benefit of long-term somatostatin analogue treatment," says researcher Giuseppe Remuzzi.

ADPKD is a progressive disease characterized by the sustained growth of fluid-filled cysts and the progressive enlargement of kidneys, often leading to the development of kidney failure in affected individuals. Although advances have been made in understanding the pathogenesis of this disease, no proven treatment exists to halt cyst growth and prevent progressive kidney dysfunction. The accelerated growth of epithelial cells and progressive growth of cysts associated with ADPKD is thought to be regulated, at least in part, by cAMP signalling. In 2005, Remuzzi and colleagues published their findings from a pilot 6-month cross-over feasibility study, which aimed to assess the effects of inhibiting cAMP production in patients with ADPKD using the somatostatin analogue octreotide long-acting release (LAR). In that study, octreotide-LAR reduced the time-dependent increase in total kidney volume by $>60 \%$ compared with placebo, prompting the researchers to perform a longer 3-year study to assess the effects of this agent in patients with ADPKD.

The current study, A Long-Acting Somatostatin on Disease Progression in Nephropathy due to Autosomal Dominant Polycystic Kidney Disease (ALADIN), enrolled adult patients with ADPKD

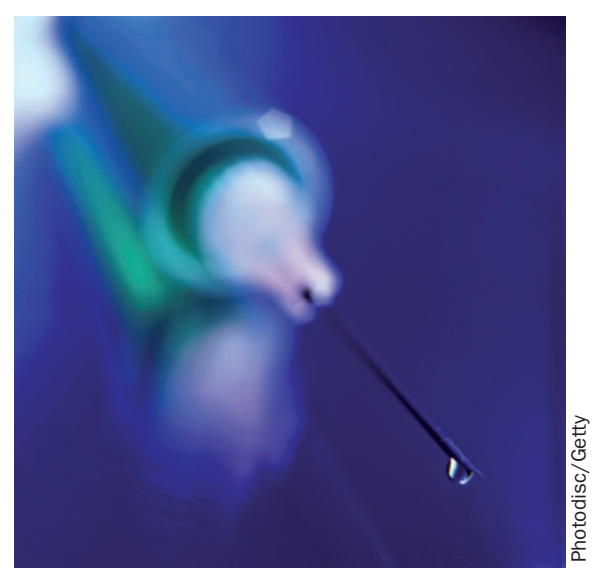

and normal renal function or mild-tomoderate renal insufficiency. 79 patients from five hospitals in Italy were randomly assigned to receive octreotide-LAR $(n=40)$ or placebo $(n=39)$ every 28 days for 3 years. "The major aims were to assess the effect of treatment on kidney and cyst growth evaluated by changes in the volume of these renal components by MRI, and on renal function decline by sequential direct measurements of true glomerular filtration rate (GFR) via the iohexol plasma clearance technique," explains Remuzzi.

At 1-year follow-up, absolute total kidney volume had increased by a significantly smaller amount in the octreotide-LAR-treated group than in the placebo group ( $46.2 \mathrm{ml}$ versus $143.7 \mathrm{ml}, P=0.032$ ). A similar trend was observed at 3 years but was not significant (220.1 $\mathrm{ml}$ versus $454.3 \mathrm{ml}, P=0.25$ ). However, the rate of total kidney volume growth was $75.4 \mathrm{ml}$ per year slower in the octreotide-LAR group than in the placebo-treated patients-a difference that was statistically significant. In addition, the percentage increase in total kidney volume was significantly smaller in the octreotide-LAR-treated group than in the placebo group at 1 year (median $0.75 \%$ versus $6.64 \%, P=0.0018$ ) and at 3 years ( $10.87 \%$ versus $19.89 \%, P=0.0416)$. The slower growth in total kidney volume in patients receiving octreotide-LAR was attributable to a slower growth in total cyst volume. Non-cyst volume was not different between the groups.

After 1 year, the researchers found that measured GFR had decreased by $10.8 \%$ from baseline in patients receiving octreotide-LAR and by $9.7 \%$ in patients receiving placebo. After this time point, however, mean GFR remained stable in the octreotide-LAR group but continued to decline in the placebo group. "The finding that after an initial reduction, GFR almost stabilized in octreotide-LARtreated patients but progressively declined in placebo-treated patients is probably the most clinically relevant finding of this study, and enables us to predict that in the long term, the somatostatin analogue will likely slow the progressive loss of renal function that can eventually result in end-stage renal disease," says Remuzzi. "We think that octreotide might blunt the compensatory hyperfiltration of glomeruli that survive the disruptive effects of uncontrolled cyst growth in patients with ADPKD, which could have significant clinical implications."

Given the lack of treatments for ADPKD, Remuzzi and colleagues believe that somatostatin analogues should be tested in larger trials that are adequately powered to test the protective effects of these agents against progression to end-stage renal disease. "In the meantime, we are working on another trial to assess the effects of octreotide-LAR in ADPKD patients with severely depressed renal function, to see whether they might also benefit from this innovative and well-tolerated treatment," says Remuzzi.

\section{Susan J. Allison}

